\title{
E-Commerce Corporations (ECCs) Internationalization: A Case Exploration: An Abstract
}

\author{
Mamoun Benmamoun, Nitish Singh, Kevin Lehnert, and Sang Bong Lee
}

\begin{abstract}
The growth of global e-commerce present significant opportunities for global expansion. Yet, it has not leveled the playing field between emerging markets e-commerce corporations (EM-ECCs) and advanced markets ECCs (AM-ECCs). While AM-ECCs have been expanding overseas with considerable success, EM-ECCs have been less disposed to internationalize and content to serve and defend their home turfs against foreign rivals wielding monopolistic advantages. Leveraging the Network, Ownership, Location and Internalization (N-OLI) framework, this paper examines the variables affecting the internationalization of AM-ECCs and EM-ECCs. We use an exploratory multiple case study approach to gain new insight into the internationalization of EM-ECCs while comparing it with the internationalization of AM-ECCs. This includes detailed analysis of two ECCs representing emerging markets and advanced markets. The findings suggest that AM ECCs, in comparison to EM ECCs, are endowed with favorable network-based advantages, firm-specific advantages, and institutional ownership advantages that make them more capable of pursuing internationalization aggressively. However, EM ECCs are induced to pursue regionally focused internationalization due, in large extent, to capital scarcity and ownership advantages emanating from knowledge of the home region.
\end{abstract}

M. Benmamoun · N. Singh $\cdot$ S. B. Lee

Saint Louis University, Saint Louis, MO, USA

e-mail: mamoun.benmamoun@slu.edu; nitish.singh@slu.edu; sangbonglee@ slu.edu

K. Lehnert (四)

Grand Valley State University, Grand Rapids, MI, USA

e-mail: lehnertk@gvsu.edu 